\title{
Future geodesic completeness of some spatially homogeneous solutions of the vacuum Einstein equations in higher dimensions
}

\author{
Arne Gödeke and Alan D. Rendall \\ Max-Planck-Institut für Gravitationsphysik \\ Albert-Einstein-Institut \\ Am Mühlenberg 1 \\ 14476 Potsdam, Germany
}

\begin{abstract}
It is known that all spatially homogeneous solutions of the vacuum Einstein equations in four dimensions which exist for an infinite proper time towards the future are future geodesically complete. This paper investigates whether the analogous statement holds in higher dimensions. A positive answer to this question is obtained for a large class of models which can be studied with the help of Kaluza-Klein reduction to solutions of the Einstein-scalar field equations in four dimensions. The proof of this result makes use of a criterion for geodesic completeness which is applicable to more general spatially homogeneous models.
\end{abstract}

\section{Introduction}

The solutions of the Einstein equations most commonly applied in cosmology are spatially homogeneous and include some that recollapse and some that expand for ever. In the former case the proper length of the timelike curves orthogonal to the hypersurfaces of homogeneity (the worldlines of comoving observers) is finite. Along a curve of this type the expansion of this congruence of curves is first positive, then zero and finally negative. This is what is meant by recollapse. In the latter case the expansion is always positive and the length of the curves orthogonal to the hypersurfaces of homogeneity is infinite towards the future. This is what is meant by saying that the solution expands for ever.

On physical grounds it is reasonable to expect that a forever expanding solution will be future geodesically complete, i.e. there should be no singularities in the future. A theorem which confirms this expectation is proved in [17] (Theorem 2.1 of that paper). In order to explain this result let $t$ be a Gaussian time coordinate based on one of the hypersurfaces of homogeneity and let trk be the mean curvature of these hypersurfaces. Then trk is a function of $t$. The 
expansion of the congruence of timelike geodesics mentioned above is $-\frac{1}{3} \operatorname{tr} k$. Define an expanding phase of the solution to be a time interval $\left(t_{1}, t_{2}\right)$ on which $\operatorname{tr} k<0$. Here $t_{1}$ and $t_{2}$ may be finite or infinite. The theorem says that, under certain assumptions, if $t_{2}<\infty$ the solution can be extended to a time interval $\left(t_{1}, t_{3}\right)$ with $t_{3}>t_{2}$. Moreover, if $t_{2}=\infty$ then the spacetime is future geodesically complete. The theorem is formulated for spatially homogeneous solutions of the Einstein equations coupled to a matter model satisfying three assumptions. Two of these are inequalities on the energy-momentum tensor, the dominant energy and non-negative sum pressures conditions. The third is a continuation property for solutions of the Einstein-matter equations. The only thing about these conditions which is important in what follows is that they are all satisfied in the case of the Einstein vacuum equations. Hence the theorem applies in the vacuum case.

In a spatially homogeneous spacetime, by definition the isometry group acts transitively on the hypersurfaces of homogeneity. In four dimensions there is a well-known classification of the possible isometry groups. Either the group can be taken to have dimension three or a higher-dimensional group is necessary. In the second of these cases there is only one possibility, which is called KantowskiSachs symmetry. In the first case any connected three-dimensional Lie group can occur. For the purposes of what follows it may be assumed without loss of generality that the spacetime is simply connected. This is because passing to the universal cover of a given spacetime has no effect on the dynamics. With this assumption the hypersurfaces of homogeneity can be identified with the group itself and the induced metric on one of these hypersurfaces with a leftinvariant metric on that group. The classification of connected and simply connected Lie groups reduces to that of the corresponding Lie algebras. The three-dimensional Lie algebras were classified by Bianchi into types I to IX. It turns out that a maximally extended vacuum spacetime of Bianchi type I-VIII expanding at some time is forever expanding while one which is of type IX or has Kantowski-Sachs symmetry recollapses [14]. The cases where recollapse takes place are precisely those where there is a spatial metric in the class whose scalar curvature $R$ is positive. That this is a necessary condition for recollapse is not hard to see. The Hamiltonian constraint on a hypersurface of homogeneity is (in the vacuum case)

$$
R-k_{a b} k^{a b}+(\operatorname{tr} k)^{2}=0
$$

where $k_{a b}$ is the second fundamental form. Recollapse means that there is a maximal hypersurface, i.e. one where $\operatorname{tr} k=0$. There $R$ is clearly non-negative. If it is zero then $k_{a b}=0$. Moreover it can be shown that if $R=0$ the induced metric of that hypersurface is flat and that the solution arising from those data is a flat spacetime where tr $k$ vanishes at all times. The latter case cannot occur in the context of spatially homogeneous cosmological models which are expanding at some time.

The aim of this paper is to investigate to what extent the results just stated for four-dimensional spatially homogeneous vacuum spacetimes extend to higher dimensions $n+1$. In particular, sufficient conditions for future geodesic com- 
pleteness are derived. Only the case where the isometry group can be taken to be of dimension $n$ is considered. This is the analogue in higher dimensions of Bianchi models. The analogue of Kantowski-Sachs models is not considered. The same argument as in four dimensions applies to show that a necessary condition for recollapse is the existence of a left-invariant metric of positive scalar curvature on the group of interest. This motivates us to restrict attention to Lie groups admitting no left-invariant metric of positive scalar curvature. In this case it has been shown that vacuum spacetimes with this type of symmetry exist for infinite Gaussian time in the future ([18], Theorem 5.3). On the other hand it turns out that the arguments used to prove geodesic completeness in the four-dimensional case do not easily generalize to higher dimensions. In what follows partial results on this question are obtained.

For a general dimension there is no known analogue of the Bianchi classification of Lie algebras. In some of the lower dimensions there are results. A review of what is known in the case of four-dimensional Lie algebras can be found in [8]. The strategy in the following is to identify some classes of Lie groups for which geodesic completeness can be proved. The main case considered is that where there is an isometry group of the form $G_{3} \times\left(\mathbb{R} \rtimes \mathbb{Z}_{2}\right)^{n-3}$ for a three-dimensional group $G_{3}$. The spatial manifold is given by the identity component of this group, $G_{3} \times \mathbb{R}^{n-3}$. The strategy for handling these groups is to use Kaluza-Klein reduction. The presence of the discrete symmetry defined by the group $\mathbb{Z}_{2}^{n-3}$ ensures that the result is the Einstein equations coupled to linear scalar fields in four dimensions. Without it a more complicated reduced system would be obtained. In the case $n=4$, for instance, it would be the Einstein-Maxwell-dilaton system. Section 2 contains the basic notation and equations, including those for the Kaluza-Klein reduction. In the third section results on the Einstein-scalar field system in four dimensions are proved. These are used in treating higherdimensional vacuum spacetimes in later sections and are also of interest in their own right. A general criterion for geodesic completeness is stated and proved in Section 4. It is then combined with the results on the scalar field to obtain the main theorem, Theorem 5 . Section 5 discusses geodesic completeness in some examples with other types of symmetry and illustrates how the results of Section 4 can be applied. Conclusions and an outlook are contained in Section 6.

This paper is based in part on the diploma thesis of the first author [7].

\section{The basic equations}

Let $G$ be a connected and simply connected Lie group and $\theta^{a}$ a basis of the space of left-invariant one-forms on $G$. Consider the metric

$$
-d t^{2}+g_{a b}(t) \theta^{a} \otimes \theta^{b}
$$

on the manifold $\left[t_{0}, \infty\right) \times G$ for some constant $t_{0}$. Let $k_{a b}$ be the components in the given basis of the second fundamental form of the level hypersurfaces of $t$. 
By the definition of the second fundamental form

$$
\partial_{t} g_{a b}=-2 k_{a b} .
$$

It will now be supposed that this metric satisfies the Einstein vacuum equations and these will be decomposed using the foliation by the level hypersurfaces of $t$. The Einstein constraint equations are

$$
\begin{aligned}
& R-k_{a b} k^{a b}+(\operatorname{tr} k)^{2}=0, \\
& \nabla_{a} k^{a}{ }_{b}=0 .
\end{aligned}
$$

The evolution equations are given by

$$
\partial_{t} k_{b}^{a}=(\operatorname{tr} k) k_{b}^{a}+R_{b}^{a} .
$$

A real number $\lambda$ is said to be an eigenvalue of $k_{a b}$ with respect to $g_{a b}$ if there is a non-zero vector $v^{a}$ which satisfies the equation $k_{a b} v^{b}=\lambda g_{a b} v^{b}$. In $n$ space dimensions there are $n$ eigenvalues of $k_{a b}$ with respect to $g_{a b}$, counting multiplicity. Call them $\lambda_{a}$. The mean curvature $\operatorname{tr} k=\sum_{a} \lambda_{a}$ never vanishes in an expanding phase. Define the generalized Kasner exponents (GKE) by $p_{a}=\frac{\lambda_{a}}{\operatorname{tr} k}$. When the Lie group $G$ is Abelian the $p_{a}$ are independent of time and the solution can be written explicitly as

$$
-d t^{2}+\sum_{a=1}^{n} t^{2 p_{a}}\left(d x^{a}\right)^{2}
$$

where $\sum_{a=1}^{n} p_{a}=1$ and $\sum_{a=1}^{n} p_{a}^{2}=1$. In the case $n=3$ this is the Kasner solution.

These equations will now be specialized to the case that the Lie group is of the form $G_{3} \times \mathbb{R}^{n-3}$ for a three-dimensional group $G_{3}$ and the left-invariant basis $\theta^{a}$ is adapted to the product decomposition. Call the basis vectors tangent to the first factor $\theta^{i}, i=1,2,3$. The basis vectors corresponding to the second factor commute and so can be chosen to be of the form $d y^{I}, I=1, \ldots, n-3$. Suppose further that the action of the group extends to an action of $G_{3} \times\left(\mathbb{R} \rtimes \mathbb{Z}_{2}\right)^{n-3}$ which preserves the hypersurfaces of constant $t$ and the product decomposition of the initial hypersurface. Then the generators of $\mathbb{Z}_{2}^{n-3}$ act by commuting reflections of $\mathbb{R}^{n-3}$. Without loss of generality they may be assumed to be given by reflections in the coordinates $y^{I}$. The invariance of the metric under these transformations implies that it can be written in the form

$$
-d t^{2}+g_{i j}(t) \theta^{i} \otimes \theta^{j}+\sum_{i} e^{2 \phi^{I}(t)}\left(d y^{I}\right)^{2}
$$

for scalar functions $\phi^{I}$. Let $\phi=\sum_{I} \phi^{I}$ and write $g_{i j}=e^{-\phi} \tilde{g}_{i j}$. Let $\tilde{t}$ be a Gaussian coordinate for the conformally rescaled metric. Then $\frac{d \tilde{t}}{d t}=e^{\frac{\phi}{2}}$. Rewriting the Einstein equations in terms of $\tilde{g}_{i j}$ and $\phi^{I}$ gives the equations

$$
\begin{aligned}
& -\tilde{k}_{i j} \tilde{k}^{i j}+(\operatorname{tr} \tilde{k})^{2}=\rho, \\
& \partial_{\tilde{t}} \tilde{k}^{i}{ }_{j}=(\operatorname{tr} \tilde{k}) \tilde{k}^{i}{ }_{j}+\tilde{R}^{i}{ }_{j}-\rho \delta_{j}^{i}, \\
& \partial_{\tilde{t}}^{2} \phi^{I}-(\operatorname{tr} \tilde{k}) \partial_{\tilde{t}} \phi^{I}=0 .
\end{aligned}
$$


Here

$$
\rho=\frac{1}{2} \sum_{I}\left(\partial_{\tilde{t}} \phi^{I}\right)^{2} .
$$

These equations are identical to the Einstein equations in four dimensions coupled to $n-3$ non-interacting massless scalar fields. For prescribed initial data it is possible to do a rotation in $\mathbb{R}^{n-3}$ so as to set all $\partial_{\tilde{t}} \phi^{I}$ except $\partial_{\tilde{t}} \phi^{1}$ to zero. By the equations of motion for the scalar fields all $\phi^{I}$ except $\phi^{1}$ will be constant during the evolution. Then only $\phi^{1}$ makes a contribution to the energymomentum tensor. The essential dynamics is that of a single scalar field. The four-dimensional metric and the scalar field $\phi^{1}$ define a five-dimensional metric. The full spacetime is obtained by taking the product of the five-dimensional metric with a flat time-independent metric.

The generalized Kasner exponents of the $(n+1)$-dimensional metric are related to the four-dimensional quantities by

$$
\begin{aligned}
& p_{a}=\frac{\tilde{\lambda}_{a}+\frac{1}{2} e^{-\frac{\phi}{2}} \partial_{t} \phi}{\operatorname{tr} \tilde{k}+\frac{1}{2} e^{-\frac{\phi}{2}} \partial_{t} \phi}, \\
& p_{I}=\frac{-e^{-\frac{\phi}{2}} \partial_{t} \phi^{I}}{\operatorname{tr} \tilde{k}+\frac{1}{2} e^{-\frac{\phi}{2}} \partial_{t} \phi} .
\end{aligned}
$$

In a spatially homogeneous situation a scalar field is equivalent to an untilted stiff fluid. Thus to understand the dynamics of the scalar field case known results for a stiff fluid can be applied. The matter variables of the two models are related by $\rho=\frac{1}{2}\left(\partial_{\tilde{t}} \phi\right)^{2}$. Note that $e^{-\frac{\phi}{2}} \partial_{t}=\partial_{\tilde{t}}$.

It was pointed out in the introduction that it is important whether a given Lie group $G$ admits left-invariant metrics with positive scalar curvature. If $G$ is a Lie group of any of the Bianchi types except IX it admits no metric of this kind. This is equivalent, for simply connected Lie groups, to the property that they are diffeomorphic to Euclidean space 2. It follows that a Lie group with topology $G \times \mathbb{R}^{n-3}$ is also homeomorphic to Euclidean space and thus also admits no left-invariant metrics of positive scalar curvature. Hence groups of this type fail the necessary condition for recollapse mentioned in the introduction. This is a sign that they are good candidates for the symmetry groups of future geodesically complete metrics.

\section{Four-dimensional models with a scalar field}

This section contains results on the late-time behaviour of solutions of the Einstein-scalar field system in four dimensions, some of which are deduced from results on untilted stiff fluids taken from the literature. When information on the dynamics of the energy density is available the asymptotics of $\phi$ can be obtained by integration. Much of the analysis is based on the Wainwright-Hsu formulation of the equations for Bianchi models [23, which will now be recalled. Since the only case of interest in the following is that of a stiff fluid the equations 
are only written down for the case where the parameter $\gamma$ in the equation of state $p=(\gamma-1) \rho$ of the fluid is equal to two. The system of evolution equations is

$$
\begin{aligned}
& N_{1}^{\prime}=\left(q-4 \Sigma_{+}\right) N_{1}, \\
& N_{2}^{\prime}=\left(q+2 \Sigma_{+}+2 \sqrt{3} \Sigma_{-}\right) N_{2}, \\
& N_{3}^{\prime}=\left(q+2 \Sigma_{+}-2 \sqrt{3} \Sigma_{-}\right) N_{3}, \\
& \Sigma_{+}^{\prime}=-(2-q) \Sigma_{+}-3 S_{+}, \\
& \Sigma_{-}^{\prime}=-(2-q) \Sigma_{-}-3 S_{-}, \\
& \Omega^{\prime}=-2(2-q) \Omega
\end{aligned}
$$

where

$$
\begin{aligned}
& q=2\left(\Omega+\Sigma_{+}^{2}+\Sigma_{-}^{2}\right) \\
& S_{+}=\frac{1}{2}\left[\left(N_{3}-N_{2}\right)^{2}-N_{1}\left(2 N_{1}-N_{2}-N_{3}\right)\right] \\
& S_{-}=\frac{1}{2}\left(N_{3}-N_{2}\right)\left(N_{1}-N_{2}-N_{3}\right) .
\end{aligned}
$$

The prime denotes $\frac{d}{d \tau}$ and $\tau$ is related to the Gaussian time $t$ by $\frac{d \tau}{d t}=-\frac{1}{3} \operatorname{tr} k$. There is also the equation which expresses the Hamiltonian constraint in these variables which is

$$
\Omega+\Sigma_{+}^{2}+\Sigma_{-}^{2}+\frac{3}{4}\left(N_{1}^{2}+N_{2}^{2}+N_{3}^{2}-2\left(N_{1} N_{2}+N_{2} N_{3}+N_{1} N_{3}\right)\right)=1 .
$$

It will not be necessary to recall the definition of all the variables in the system here. What is important is that $\Sigma_{+}$and $\Sigma_{-}$are linear combinations of the generalized Kasner exponents which are linearly independent. This means that if $\Sigma_{+}$and $\Sigma_{-}$tend to finite limits as $t \rightarrow \infty$ the same is true of $p_{1}, p_{2}$ and $p_{3}$. The density parameter $\Omega$ is equal to $\frac{3 \rho}{(\operatorname{tr} k)^{2}}$. The mean curvature can be recovered from a solution of the Wainwright-Hsu system using the equation

$$
(\operatorname{tr} k)^{\prime}=-(1+q)(\operatorname{tr} k) .
$$

Theorem 1 Let $(M, g)$ be a solution of the Einstein equations coupled to a scalar field $\phi$ in four dimensions with symmetry of a Bianchi type other than $I X$ or $V I_{-\frac{1}{9}}$. If the solution is expanding at some time and the time interval of definition of the solution is maximal then the upper limit of the interval is infinity and the generalized Kasner exponents and the density parameter $\Omega$ converge to limits as $t \rightarrow \infty$.

Proof Bianchi type I solutions are defined by the condition $N_{1}=N_{2}=N_{3}=0$. It follows from the Hamiltonian constraint that $q=2$ and that $\Sigma_{+}, \Sigma_{-}$and $\Omega$ are constant. That the conclusion of the theorem holds for Bianchi type II solutions, which are defined by the conditions $N_{2}=N_{3}=0$ and $N_{1} \neq 0$, was shown in Lemma 7.1 of [19]. Bianchi type $\mathrm{VI}_{0}$ solutions are defined by the 
conditions $N_{1}=0, N_{2}>0$ and $N_{3}<0$. The evolution equation for $\Sigma_{+}$reduces to

$$
\Sigma_{+}^{\prime}=-(2-q)\left(\Sigma_{+}+1\right)
$$

It follows from the Hamiltonian constraint that $q<2$. This in turn implies that $\Sigma_{+}+1$ is strictly positive and that $\Sigma_{+}$is strictly decreasing. Applying the monotonicity principle to $\Sigma_{+}$shows that there can be no $\omega$-limit point with $q<2$. Since the solution stays in a compact region it follows that $q \rightarrow 2$ as $\tau \rightarrow \infty$ and that $N_{1}$ and $N_{2}$ tend to zero. Since $\Sigma_{+}$and $\Omega$ are monotone and bounded both of them tend to limits as $\tau \rightarrow \infty$. By the Hamiltonian constraint the same is true of $\Sigma_{-}$. Bianchi type $\mathrm{VII}_{0}$ solutions are defined by the conditions $N_{1}=0, N_{2}>0$ and $N_{3}>0$. Locally rotationally symmetric solutions of type $\mathrm{VII}_{0}$ are defined by the additional conditions $N_{2}=N_{3}$ and $\Sigma_{-}=0$. In this case $S_{+}, S_{1}$ are zero and $q=2$. Hence $\Omega$ and $\Sigma_{+}$are constant. The proof for solutions of type $\mathrm{VII}_{0}$ which are not locally rotationally symmetric results from a small modification of the proof of Proposition 5 of [20, which treats the corresponding question in the vacuum case. First it can be shown by an argument similar to that used for type $\mathrm{VI}_{0}$ case that $\Omega$ and $\Sigma_{+}$are strictly monotone. This makes use of the equation

$$
\left(N_{2}-N_{3}\right)^{\prime}=\left(q+2 \Sigma_{+}\right)\left(N_{2}-N_{3}\right)+2 \sqrt{3} \Sigma_{-}\left(N_{2}+N_{3}\right)
$$

to treat the points where $\Sigma_{+}^{\prime}$ vanishes. Since $\Omega$ and $\Sigma_{+}$are also bounded they must converge as $t \rightarrow \infty$. It is not known a priori that the whole solution is bounded and so it may not have an $\omega$-limit point. Suppose that it does have such a point. Using the monotonicity principle it can be shown that if $\tau_{n}$ is a sequence of times along which the solution converges then either $\left(\Sigma_{-}^{2}+\left(N_{2}-N_{3}\right)^{2}\right)\left(\tau_{n}\right)$ tends to zero or $N_{1}+N_{2}$ does so. It can then be concluded as in 20, using the monotone function $Z_{-1}$ of that paper that $\Sigma_{-} \rightarrow 0$ as $\tau \rightarrow \infty$. Note that $Z_{-1}$ is monotone in the case with matter (see [20], Lemma 10.1). It remains to treat the case in which the solution has no $\omega$ limit point. Then $N_{1}$ and $N_{2}$ must tend to infinity and it can be assumed without loss of generality that the limit of $\Omega+\Sigma_{+}^{2}$ is less than one. In particular this means that the limit of $\Sigma_{+}$cannot be minus one. Hence, by (26) the quantity $2-q$ must be in $L^{1}$. It can, however, be shown by a slight modification of an argument of Ringström (20, proof of Proposition 5) that $2-q$ is not in $L^{1}$. That argument uses two quantities called $x$ and $y$ and the necessary modification is to replace them by

$$
\begin{aligned}
& x=\frac{\Sigma_{-}}{\left(1-\Sigma_{+}^{2}-\Omega\right)^{\frac{1}{2}}}, \\
& y=\frac{\sqrt{3}}{2} \frac{N_{2}-N_{3}}{\left(1-\Sigma_{+}^{2}-\Omega\right)^{\frac{1}{2}}} .
\end{aligned}
$$

Bianchi type VIII solutions are defined by the conditions $N_{1}<0, N_{2}>0$ and $N_{3}<0$. The result of the theorem in this case follows from Theorem 3.1 and Corollary 3.1 of $[12$. 
For models of Bianchi class B it follows from Proposition 5.3 of [1] that the $\omega$-limit set of any solution lies in a set $\overline{\mathcal{L}}_{k}$. The parameter $k$ distinguishes the different Bianchi types and is equal to $h^{-1}$ in types $\mathrm{VI}_{h}$ and $\mathrm{VII}_{h}$. For each value of $k$, the set $\overline{\mathcal{L}}_{k}$ consists of equilibrium solutions. It is an open curve $\mathcal{L}_{k}$ together with its two endpoints. The $\omega$-limit set of a solution is in fact a single point of $\overline{\mathcal{L}}_{k}$. This can be seen as follows. A generic point of the curve is transversely hyperbolic and so if it belongs to the $\omega$-limit set no other point can. The remaining points form a discrete set and so by connectedness of the $\omega$-limit set it cannot contain more than one of them.

The fact that Bianchi type IX is excluded in the hypotheses of this theorem is essential. For the metric product of a four-dimensional solution of Bianchi type IX with the real line fails to exist globally towards the future. On the other hand there is no reason to expect that type $\mathrm{VI}_{-\frac{1}{9}}$ has to be excluded. It appears nevertheless that up to now no proof is available in that case.

Some more precise information will now be given on the asymptotics of scalar field models. Let $\mathcal{D}$ be the disk in the space with coordinates $\left(\Sigma_{+}, \Sigma_{-}, N_{1}, N_{2}, N_{3}\right)$ defined by the conditions that the $N_{i}$ are all zero and $\Sigma_{+}^{2}+\Sigma_{-}^{2}<1$. Let $\mathcal{L}$ be the line defined by $N_{1}=0, N_{2}=N_{3}>0, \Sigma_{+}=-1$ and $\Sigma_{-}=0$.

Theorem 2 Let $(M, g)$ be a solution of the Einstein equations coupled to a scalar field in four dimensions with symmetry of Bianchi class A. If the Bianchi type is I, II or VI $I_{0}$ then the variables in the Wainwright-Hsu system converge to limits as $\tau \rightarrow \infty$. If the Bianchi type is VIII the $\omega$-limit set of each solution is empty. In type VII $I_{0}$ both types of behaviour occur. The sets of points which occur as $\omega$-limit points is the disk $\mathcal{D}$ in type $I$, the open subset of $\mathcal{D}$ defined by the inequality $\Sigma_{+}>\frac{1}{2}$ in type II, the point of $\overline{\mathcal{D}}$ with $\left(\Sigma_{+}, \Sigma_{-}\right)=(-1,0)$ in type $V I_{0}$ and $\mathcal{L}$ in LRS type VII . The limit of $\Omega$ is non-zero in types $I$ and $I I$ and zero in types $V I_{0}$ and VIII.

Proof The Bianchi type I solutions of the Wainwright-Hsu system with a stiff fluid are time independent and are exactly the points of $\mathcal{D}$. It follows from Theorem 1 that any solution of type II converges to a point of $\mathcal{D}$ as $\tau \rightarrow \infty$. It is a consequence of the evolution equation for $N_{1}$ that this point must satisfy $\Sigma_{+} \geq 0$. A more detailed analysis reveals that this inequality is strict. To see this note that all terms on the right hand side of the equations for Bianchi type II solutions have a common factor $N_{1}$. Omitting this factor leads to a new system whose integral curves are the same as those of the original system in the region where $N_{1} \neq 0$. The new system is

$$
\begin{aligned}
& \dot{N}_{1}=2\left(1-2 \Sigma_{+}\right)-\frac{3}{2} N_{1}^{2}, \\
& \dot{\Sigma}_{+}=3\left(1-\frac{1}{2} \Sigma_{+}\right) N_{1}, \\
& \dot{\Sigma}_{-}=-\frac{3}{2} N_{1} \Sigma_{-} .
\end{aligned}
$$


This system has a line of stationary solutions defined by the conditions $N_{1}=0$ and $\Sigma_{+}=\frac{1}{2}$. Linearizing the system about one of these points reveals that it has the eigenvalues $0, \pm 3 i$. Because of the reduction theorem (cf. [18], section 5.6) the qualitative behaviour of the solutions can be determined by analysing the dynamics on a centre manifold. The system on the centre manifold is such that no solution can converge to the stationary point while staying in the region $N_{1}>0$ (cf. [16], p. 144, Theorem 5) and this implies the desired result. Any point of $\mathcal{D}$ with $\Sigma_{0}>\frac{1}{2}$ occurs as the $\omega$-limit point of a solution of type II since the linearization there has a negative eigenvalue. By Theorem 1 any solution of type $\mathrm{VI}_{0}$ converges to a point of $\overline{\mathcal{D}}$. As a consequence of the evolution equations for $N_{2}$ and $N_{3}$ this can only be the point where $\Sigma_{+}=-1$. In particular $\Omega \rightarrow 0$ and the solution looks asymptotically like a vacuum solution. Any locally rotationally symmetric solution of type $\mathrm{VII}_{0}$ has $\Sigma_{-}=0, N_{2}=N_{3}$ and a constant value of $\Sigma_{+}$. As $\tau \rightarrow \infty$ the quantity $N_{2}$ tends to infinity. For type $\mathrm{VII}_{0}$ solutions which are not LRS it has already been shown that $\Sigma_{+}^{2}+\Omega \rightarrow 1$ and that $\Sigma_{-}$and $N_{2}-N_{3}$ tend to zero. If the solution has no $\omega$-limit point then $\Omega \rightarrow 0$ as $\tau \rightarrow \infty$ and $\Sigma_{+} \rightarrow 1$. For general type VIII it is shown in [12] that $\Omega \rightarrow 0$ and $N_{+} \rightarrow \infty$.

\section{A criterion for geodesic completeness}

The aim of this section is to prove the following criterion for geodesic completeness. The general set-up is a spatially homogeneous spacetime defined by a one-parameter family $g_{a b}(t)$ of left-invariant metrics on a Lie group $G$ of dimension $n$.

Theorem 3 Let $(M, g)$ be a locally spatially homogeneous vacuum spacetime of dimension $n+1<10$ whose symmetry is defined by a Lie group $G$ which admits no left-invariant metrics of positive scalar curvature. If for some $t_{0}$ the generalized Kasner exponents satisfy

$$
\sup _{t \geq t_{0}} \min _{a} p_{a}(t)-\inf _{t \geq t_{0}} \min _{a} p_{a}(t) \leq \frac{9-n}{4 n}
$$

then the spacetime is geodesically complete.

Proof Since the scalar curvature is non-positive it follows from the Hamiltonian constraint that $k_{a b} k^{a b} \leq(\operatorname{tr} k)^{2}$ and hence that $\sum_{a} p_{a}^{2} \leq 1$. The minimum of $\sum_{a} p_{a}^{2}$ subject to the constraint $\sum_{a} p_{a}=1$ occurs when all $p_{a}$ are equal, as follows from the method of Lagrange multipliers. Hence

$$
k_{a b} k^{a b} \geq \frac{(\operatorname{tr} k)^{2}}{n} .
$$

The minimum possible value of $p_{a}$ is $-\frac{n-2}{n}$, as has been proved in [18, p. 96. By symmetry this is also the minimum value of any other $p_{a}$. It can be concluded 
that the maximum eigenvalue $\lambda_{\max }$ satisfies

$$
\frac{1}{n} \operatorname{tr} k \leq \lambda_{\max } \leq-\frac{n-2}{n} \operatorname{tr} k .
$$

If the value of $\lambda_{\max }$ is fixed the inequality (34) can be improved. Assume that $\lambda_{\max }=\lambda_{1}$. Using Lagrange multipliers again it can be seen that the minimum of $k_{a b} k^{a b}$ is attained when all $\lambda_{a}$ other than $\lambda_{1}$ are equal. Hence the lower bound for $k_{a b} k^{a b}$ is got by setting

$$
\lambda_{2}=\ldots=\lambda_{n}=\frac{\operatorname{tr} k-\lambda_{\max }}{n-1} .
$$

It follows that $k_{a b} k^{a b}$ is bounded below by

$$
\begin{aligned}
& \lambda_{\max }^{2}+\frac{\left(\operatorname{tr} k-\lambda_{\max }\right)^{2}}{n-1} \\
& =(\operatorname{tr} k)^{2}\left(n p_{\min }^{2}-2 p_{\min }+1\right)(n-1)^{-1} \\
& =(\operatorname{tr} k)^{2} P\left(p_{\min }\right) .
\end{aligned}
$$

The function $P$ is positive and decreasing on $\left[-\frac{n-2}{n}, \frac{1}{n}\right]$. Let $p_{0}$ be the supremum in $t$ of $p_{\min }(t)$. Then by definition $p_{\min }(t) \leq p_{0}$ for all $t$. On the other hand the hypotheses of the theorem imply that $p_{\min }(t) \geq p_{0}-\Delta$ where $\Delta=p_{0}-\inf _{t \geq t_{0}} \min _{a} p_{a}(t)$. Assume that $p_{0}-\Delta<0$. (Geodesic completeness in the case $p_{0} \geq \Delta$ is treated in Theorem 4.) Now

$$
\partial_{t}(\operatorname{tr} k)=k_{a b} k^{a b} \geq P\left(p_{0}\right)(\operatorname{tr} k)^{2} .
$$

Integrating this in $t$ gives

$$
(\operatorname{tr} k)(t) \geq \frac{-P\left(p_{0}\right)^{-1}}{C+t}
$$

for a constant $C$ depending on $t_{0}$ and $\operatorname{tr} k\left(t_{0}\right)$. Let $q^{a}$ be the projection of the tangent vector to a causal geodesic onto the hypersurfaces of constant $t$. Then

$$
\frac{d}{d t}\left(g_{a b} q^{a} q^{b}\right)=2 k_{a b} q^{a} q^{b} \leq 2\left(p_{0}-\Delta\right)(\operatorname{tr} k) g_{a b} q^{a} q^{b} .
$$

Replacing trk in this inequality using (39) and integrating gives

$$
\left(g_{a b} q^{a} q^{b}\right)^{-\frac{1}{2}} \geq C(1+t)^{-\lambda}
$$

where $\lambda=\frac{\Delta-p_{0}}{P\left(p_{0}\right)}$. The affine parameter length of a causal geodesic up to time $\tau$ is given by

$$
\int_{t_{o}}^{\tau}\left(\epsilon+g_{a b} q^{a} q^{b}\right)^{-\frac{1}{2}} d t
$$

where $\epsilon$ is one for timelike geodesics parametrized by arc length and zero for null geodesics. This integral diverges provided $\lambda \leq 1$. It remains to show that this follows from the inequality $\Delta \leq \frac{9-n}{4 n}$. Now

$$
\frac{\Delta-p_{0}}{P\left(p_{0}\right)} \leq 1
$$


is equivalent to

$$
p_{0}^{2}+\frac{n-3}{n} p_{0}+\frac{1}{n}-\frac{n-1}{n} \Delta \geq 0
$$

The expression on the left hand side of this inequality has its minimum at $p_{0}=-\frac{n-3}{2 n}$. Its value there is

$$
\frac{n-1}{n}\left(\frac{9-n}{4 n}-\Delta\right) .
$$

This completes the proof of the theorem.

For simplicity this theorem has been stated for the vacuum case only. In fact the theorem, and its proof, generalize straightforwardly to the case where matter is present provided the matter satisfies the dominant and strong energy conditions and has reasonable evolution properties. The precise formulation of the last property is the matter continuation criterion (MCC) introduced in [18]. A variant of Theorem 3 is given by

Theorem 4 Let $(M, g)$ be a locally spatially homogeneous vacuum spacetime of dimension $n+1$ whose symmetry is defined by a Lie group $G$ which admits no left-invariant metrics of positive scalar curvature. If for some $t_{0}$ the generalized Kasner exponents satisfy $p_{a} \geq-\frac{1}{n}$ then the spacetime is geodesically complete.

Proof The proof proceeds in a similar way to that of the previous theorem. This time the inequality $\partial_{t}(\operatorname{tr} k) \geq \frac{(\operatorname{tr} k)^{2}}{n}$ implies that

$$
(\operatorname{tr} k)(t) \geq-\frac{n}{C+t}
$$

and that

$$
\left(g_{a b} q^{a} q^{b}\right)^{-\frac{1}{2}} \geq C(1+t)^{-\lambda}
$$

This completes the proof.

This theorem also generalizes easily to the case with matter satisfying the DEC, SEC and MCC. In the case $n=3$ the hypothesis on the $p_{a}$ is satisfied identically.

Next a large class of Lie groups will be exhibited for which the assumptions of Theorem 3 are satisfied. They are obtained using products of three-dimensional Lie groups with Abelian groups of arbitrary dimensions.

Theorem 5 Let $(M, g)$ be a spatially homogeneous solution of the vacuum Einstein equations in $n+1$ dimensions with $3 \leq n<9$. Suppose that the group defining the homogeneity is of the form $G_{3} \times\left(\mathbb{R} \rtimes \mathbb{Z}_{2}\right)^{n-3}$ for some three-dimensional Lie group $G$ whose Bianchi type is neither $I X$ or $V I_{-\frac{1}{9}}$. If the solution is maximally extended to the future then it is geodesically complete.

Proof Under the hypotheses of this theorem Kaluza-Klein reduction can be used to relate the generalized Kasner exponents of the higher dimensional vacuum model to the generalized Kasner exponents of the reduced Einstein-scalar 
field model in four dimensions. By Theorem 1 and the formulae (13) and (14) it follows that the generalized Kasner exponents of the $(n+1)$-dimensional spacetime converge as $t \rightarrow \infty$. This conclusion makes use of the fact that, since $\frac{2 \Omega}{3}=\left(\frac{\partial_{\tilde{t}} \phi}{\operatorname{tr} \tilde{k}}\right)^{2}$, the convergence of $\frac{\partial_{\tilde{t}} \phi}{\operatorname{tr} \tilde{k}}$ is equivalent to that of $\Omega$. Hence $p_{0}=0$ and the hypotheses of Theorem 3 are satisfied.

\section{$5 \quad$ Further examples}

In the previous section geodesic completeness was proved for models based on Lie groups belonging to a certain class not admitting metrics of positive scalar curvature. To go further it is necessary to enter into the structure of more general Lie algebras. (A good general reference for the theory of Lie groups and Lie algebras is 22.) For this some definitions are necessary. If $L$ is a Lie algebra denote by $[L, L]$ the subalgebra generated by the elements of $L$ of the form $[x, y]$ for $x$ and $y$ in $L$. Define $L^{(n)}$ recursively by $L^{(n+1)}=\left[L^{(n)}, L^{(n)}\right]$ and $L^{(1)}=L$. If $L^{(n)}=\{0\}$ for some $n$ then $L$ is called solvable. The radical $R$ of a Lie algebra $L$ is an ideal of $L$ which is solvable and which contains all other solvable ideals. A Levi subalgebra is a subalgebra which, as a vector space, is complementary to the radical. It is semisimple which means that it has no nontrivial solvable ideals. A derivation of $L$ is a linear mapping $D$ which satisfies $D([x, y])=[D x, y]+[x, D y]$ for all $x$ and $y$. A particular type of derivations are the inner derivations which are of the form $[x$,$] for some element x$ of the algebra. Let $L_{1}$ and $L_{2}$ be two Lie algebras. Denote by $\operatorname{Der} L_{2}$ the vector space of all derivations of $L_{2}$. It is closed under commutators and in this way acquires the structure of a Lie algebra. Let $\phi$ be a Lie algebra homomorphism from $L_{1}$ to Der $L_{2}$. A Lie algebra $L$ can be defined as the direct sum of the underlying vector spaces of $L_{1}$ and $L_{2}$ with the Lie bracket defined by

$$
\left[\left(x_{i}, y_{1}\right),\left(x_{2}, y_{2}\right)\right]=\left(\left[x_{1}, x_{2}\right],\left[y_{1}, y_{2}\right]+\phi\left(x_{1}\right)\left(y_{2}\right)-\phi\left(x_{2}\right)\left(y_{1}\right)\right) .
$$

The Lie algebra $L$ is called the semidirect sum of $L_{1}$ and $L_{2}$. The Levi-Malcev theorem says that any finite-dimensional Lie algebra can be written as a semidirect sum of its radical $R$ with a semisimple Lie algebra.

Consider now the case of four-dimensional Lie algebras. It is known that the only semisimple Lie algebras of dimension no greater than four are $s u(2)$ and $\operatorname{sl}(2, \mathbb{R})$. It follows that the only four-dimensional Lie algebras which are not solvable are semidirect sums of the real numbers with $\operatorname{su}(2)$ and $\operatorname{sl}(2, R)$. In fact the semidirect sum of the real numbers with a semisimple Lie algebra is isomorphic to a direct sum. To see this note that every derivation of a semisimple Lie algebra is inner. Hence the mapping $\phi$ defining the semidirect sum must be of the form $\phi(x)=\phi_{S}(x)\left[y_{0},\right]$ for a linear map $\phi_{S}$ from $L_{1}$ to $\mathbb{R}$ and some $y_{0} \in L_{2}$. It then follows that the linear map $\psi$ from $L$ to itself defined by

$$
\psi(x, y)=\left(x, y+\phi_{S}(x) y_{0}\right)
$$

is an isomorphism from the given Lie algebra to the direct sum. The group $S U(2) \times \mathbb{R}$ admits left-invariant metrics of positive scalar curvature because 
$S U(2)$ does. On the other hand it is well-known that the simply connected Lie group with Lie algebra $s l(2, \mathbb{R})$ is diffeomorphic to $\mathbb{R}^{3}$. Hence the group corresponding to the Lie algebra $s l(2, \mathbb{R}) \oplus \mathbb{R}$ is diffeomorphic to $\mathbb{R}^{4}$. Any simply connected solvable Lie group is diffeomorphic to $\mathbb{R}^{n}$ for some $n$ and hence admits no metric of positive scalar curvature. Putting these facts together it can be concluded that the only connected and simply connected four-dimensional Lie group which admits a metric of positive scalar curvature is $S U(2) \times \mathbb{R}$. This fact was noted in the thesis of Hervik [9], who refers to a paper of Patera et. al. [15]. In the latter a list of Lie algebras of dimensions 4 and 5 is given which are not direct sums and they are all stated to be solvable. It may be noted in passing that on the basis of this the only types for which recollapse can occur in five space dimensions are $s u(2) \oplus \mathbb{R}^{2}$ and $s u(2) \oplus A_{2,1}$. A general criterion for the existence of left-invariant metrics of positive scalar curvature in any dimension has been given in [10. The facts just mentioned suggest the following conjecture:

Conjecture Let $G$ be a connected and simply connected four-dimensional Lie group which is not isomorphic to $S U(2) \times \mathbb{R}$. Then any spatially homogeneous solution of the vacuum Einstein equations with this symmetry group which is expanding at some time is future geodesically complete.

Theorem 5 proves this conjecture for Lie algebras which are the direct sum of a three-dimensional Lie algebra and the real numbers and metrics which have an additional discrete symmetry. Another case where something is known is that where the four-dimensional algebra is a direct sum of two non-Abelian subalgebras [1]. In the notation of [15] and [8] this is the case $A_{2,1} \oplus A_{2,1}$. The metrics covered by the theorem of [1] are not the most general left-invariant metrics. They have a six-dimensional symmetry group. By Theorem 3.1 of [1] the quantities $P$ and $Q$ converge to positive limits as $t \rightarrow \infty$ and these are essentially the generalized Kasner exponents. Hence Theorem 4 above applies. Similar results are obtained in higher dimensions. These apply for example to Lie algebras of the form $A_{2,1} \oplus \mathbb{R}^{n}$ for any $n$. The limits of the generalized Kasner exponents are non-negative. It is once again the case that only metrics with additional symmetry are included.

The Lie algebras which define Bianchi models of class A are those which are unimodular, i.e. those for which the trace of the structure constants is zero. A four-dimensional Lie algebra which is the direct sum of a three-dimensional algebra with the real numbers is unimodular if and only if the three-dimensional Lie algebra is of class A. The unimodular four-dimensional Lie algebras which are not of this form can be read off from Table 1 in [8]. In the notation used there they are $A_{4,1}, A_{4,2}^{-2}, A_{4,5}^{p q}$ for $p+q=-1, A_{4,6}^{p q}$ for $p+2 q=0, A_{4,8}$ and $A_{4,10}$. Some explicit solutions are known. In the cases $A_{4,2}^{-2}$ and $A_{4,5}^{p q}$ explicit diagonal solutions are given in [5]. In both cases the generalized Kasner exponents converge to $(1,0,0,0)$ and thus these solutions are future geodesically complete by Theorem 4. In the case $A_{4,8}$ a solution which is diagonal and selfsimilar is given in [3. Its generalized Kasner exponents are time-independent. They are equal to $\left(\frac{3}{4},-\frac{1}{4}, \frac{1}{4}, \frac{1}{4}\right)$. Theorem 4 applies to this solution and it is 
interesting to note that this is a borderline case for that theorem. In the case $A_{4,10}$ an explicit solution is mentioned in 3 but the authors note that it has a higher dimensional symmetry group and can also be considered as corresponding to the Lie algebra which is the direct sum of the Bianchi type II Lie algebra with the real numbers. It admits a reflection symmetry of the type which makes it belong to the class covered by Theorem 5 .

There is a way of fitting many of these Lie algebras in a common geometrical framework. In [17] the notion of local $U(1) \times U(1)$ symmetry was introduced and it was shown how the Bianchi types I, II, $\mathrm{VI}_{0}$ and $\mathrm{VII}_{0}$ can be identified among spacetimes with two commuting spacelike local Killing vectors and a global topology which is a bundle over the circle with fibre $T^{2}$. The different Bianchi types are distinguished by the algebraic structure of a certain $2 \times 2$ matrix with unit determinant. If both eigenvalues are real with unit modulus and the matrix is diagonalizable then type I is obtained. A matrix which is not diagonalizable gives rise to type II. When the eigenvalues are real and distinct type $\mathrm{VI}_{0}$ results. Finally type $\mathrm{VII}_{0}$ is obtained when the eigenvalues are imaginary. The same analysis can be repeated for one dimension higher, although it does not cover all relevant algebras. In this case local $U(1) \times U(1) \times U(1)$ symmetry is the basic input and different cases are classified by the algebraic properties of a $3 \times 3$ matrix with unit determinant. If there is a real eigenvalue with unit modulus and the corresponding Jordan block is one by one then a case is obtained where the Lie algebra is the direct sum of one of the algebras seen in the three-dimensional case with the real numbers. The only other case where all eigenvalues have are real with unit modulus is that of a $3 \times 3$ Jordan block. This gives the algebra $A_{4,1}$. When the eigenvalues are real and all distinct type $A_{4,5}^{p q}$ results. In the case that there is pair of non-real eigenvlaues the Lie algebra which arises is $A_{4,6}^{p q}$. If there is a non-trivial Jordan block corresponding to a double eigenvalue then $A_{4,2}^{-2}$ occur: 1 . This construction does not apply to types $A_{4,8}$ and $A_{4,10}$ since it can be shown straightforwardly that neither of these has a three-dimensional Abelian subalgebra. This point of view shows that in all the cases where the Lie algebra has a three-dimensional Abelian subalgebra the group can be made to act on the universal cover of a spacetime with a compact Cauchy surface by isometries of the pull-back of the spacetime metric. The topology of the Cauchy surface is that of a bundle over $S^{1}$ with fibre $T^{3}$.

A convenient feature of vacuum Bianchi models of class A is that initial data whose components in a suitable basis are diagonal lead to solutions which are also diagonal in that basis. Call a basis of this kind a canonical basis. It is moreover the case that for any initial data of Bianchi class A there is a canonical basis in which it is diagonal. These facts together mean that in order to understand the dynamics of vacuum models of Bianchi class A it is enough to understand the dynamics of diagonal models. The fact that models which are initially diagonal remain diagonal can be proved using automorphisms of the Lie algebras involved. For convenience let the linear transformation which

\footnotetext{
${ }^{1}$ Remarks related to the material of this paragraph are contained in an unpublished manuscript of S. Hervik
} 
multiplies $e_{i}$ by $(-1)^{a_{i}}, a_{i} \in\{0,1\}$, be denoted by $\left(a_{1} a_{2} a_{3}\right)$. In a canonical basis the transformations (110), (101) and (011) are automorphisms. In other words, they leave the structure constants invariant. Data which are diagonal in the given basis are invariant under these transformations. Because the structure constants are invariant the Einstein evolution equations are also invariant under these transformations. It follows from the unique determination of solutions by data that invariant data lead to invariant solutions. A solution which is invariant under all these transformations is diagonal. It would be nice if this argument could be generalized to the case of unimodular Lie algebras in higher dimensions. This is no problem if the Lie algebra is a direct sum of a three-dimensional Lie algebra with the real numbers. For in that case the automorphisms of the three-dimensional case can be trivially extended to get (in a hopefully obvious notation) the automorphisms (1100), (1010) and (0110). For the other Lie algebras this method has very limited success, at least if the bases in which the Lie algebras are presented in [8] are taken as candidates for a canonical basis. The only case in which there are enough automorphisms obtained by changing the signs of some of the basis vectors to make the argument go through is $A_{4,5}^{p q}$. For that Lie algebra any automorphism must fix $e_{4}$ but the signs of the other basis vectors can be changed independently. In other words it is possible to use the automorphisms (1000), (0100) and (0010). The Wainwright-Hsu system has been very useful for the study of Bianchi class A models. Its simplicity relies on the diagonalization property and so it may be that nothing of comparable power is available for general unimodular Lie algebras in any higher dimension.

The theme of the present paper is the late-time behaviour of cosmological models but the behaviour near the initial singularity is also of great interest. For the latter subject the issue of diagonalization plays a very important role. In spacetime dimension four it is believed that generic spatially homogeneous solutions of the Einstein vacuum equations show oscillatory (Mixmaster) behaviour near the initial singularity and this has been proved rigorously in some cases. (For Bianchi type IX see [19].) In $4+1$ dimensions it seems that this is still true but that restricting to the special case of diagonal models removes the generic oscillations [6]. In particular, generic oscillations can occur for the group $S U(2) \times \mathbb{R}$ due to the influence of the non-diagonal degrees of freedom. The situation is similar in dimensions up to $9+1$ but beyond that the oscillations are no longer generic. For some rigorous results on this see [4].

\section{Conclusions and outlook}

In the preceding sections it has been proved that spatially homogeneous solutions of the Einstein equations in various dimensions with symmetries defined by a large class of Lie groups are geodesically complete in the future. The case considered in most detail is that of spacetime dimension five. Even there it is to be hoped that something much more general can be proved. A suggestion for a generalization of this kind is formulated as a conjecture in Section 5. Making a corresponding conjecture in general dimensions is dependent on 
a sufficently good understanding of the structure of general Lie algebras. A guide in formulating the conjecture was to restrict to those Lie groups which do not admit a metric of positive scalar curvature. Another direction of possible generalization is to consider spatially homogeneous spacetimes where the Lie group defining the symmetry is necessarily of a dimension higher than the space dimension. These generalize the Kantowski-Sachs models in spacetime dimension four. In five spacetime dimensions the list of possibilities is as follows [8]: the four-sphere $S^{4}$ with symmetry group $S O(4)$, the complex projective plane $\mathbb{C P}^{2}$ with an eight-dimensional symmetry group, $S^{2} \times S^{2}$ with a six-dimensional symmetry group, $S^{2} \times \mathbb{R}^{2}$ and $S^{2} \times H^{2}$ with five-dimensional symmetry groups. All of these examples admit an invariant metric with positive scalar curvature and so are not promising candidates for allowing spacetimes which are future geodesically complete. The theorem of Bérard-Bergery 2 mentioned at the end of Section 2 extends to homogeneous spaces which are more general than Lie groups and may be helpful in investigating generalizations of Kantowski-Sachs models in higher dimensions.

In the previous sections the cosmological constant $\Lambda$ has always been assumed to vanish. It is likely that the theorem of Wald 24] on the late-time asymptotics of spatially homogeneous cosmological models with $\Lambda>0$ extends to arbitrary dimensions with the essential assumption (in the vacuum case) being the absence of metrics of positive scalar curvature. Combining this with the ideas in [13] should give geodesic completeness. These ideas have, however, not yet been worked out in detail.

There are very few results on future geodesic completeness for inhomogeneous spacetimes which do not rely on choosing initial data which are close to those of a known solution (small data results). This applies even in the a priori simpler case $\Lambda>0$. In fact the only result of this kind for vacuum spacetimes is that of Ringström 21. It could be that obtaining a better understanding of geodesic completeness for homogeneous models in higher dimensions would provide new ideas for understanding the inhomogeneous case in spacetime dimension four.

It is interesting to ask what happens when there is a metric of positive scalar curvature. Is there always recollapse in that case? It seems that very little is known about this in spacetime dimensions greater than four. There are also few results available for inhomogeneous solutions in four dimensions. Perhaps a better understanding of the homogeneous case in higher dimensions could help to change this. In particular, higher dimensions could be a good place to look for a counterexample.

The long-time behaviour of solutions of the vacuum Einstein equations in higher dimensions gives rise to a rich variety of problems concerning the dynamics of the gravitational field. In this paper we have obtained theorems on some of the simpler of these, while pointing out promising directions for future research. We have also emphasized that one motivation for studying these questions is the insights they may bring for the Einstein-matter equations in four dimensions. 


\section{References}

[1] Andersson, L. and Heinzle, M. 2007 Eternal acceleration from M-theory. Adv. Theor. Math. Phys. 11, 371-398.

[2] Bérard-Bergery, L. 1978 Sur la courbure des métriques riemanniennes invariantes des groupes de Lie et des espaces homogènes. Ann. Sci. Ecole Norm. Sup. (4) 11, 543-576.

[3] Christodoulakis, T., Hervik, S. and Papadopoulos, G. O. 2004 Essential constants for spatially homogeneous Ricci-flat manifolds of dimension $4+1$. J. Phys. A37, 4039-4058.

[4] Damour, T., Henneaux, M., Rendall, A. D. and Weaver, M. 2002 Kasnerlike behaviour for subcritical Einstein-matter systems. Ann. H. Poincaré 3, 1049-1111.

[5] Demaret, J. and Hanquin, J.-L. 1985 Anisotropic Kaluza-Klein cosmologies. Phys. Rev. D31, 258-261.

[6] Demaret, J., de Rop, Y. and Henneaux, M. Are Kaluza-Klein models of the universe chaotic? Int. J. Theor. Phys. 28, 1067-1079.

[7] Gödeke, A. 2009 Long-term behavior of higher dimensional spatially homogeneous cosmological models. Diploma thesis, Free University, Berlin.

[8] Hervik, S. 2002 Multidimensional cosmology: spatially homogeneous models of dimension 4+1. Class. Quantum Grav. 19, 5409-5428.

[9] Hervik, S. 2004 Mathematical cosmology: Bianchi models, asymptotics and extra dimensions. D. Phil. Thesis, University of Cambridge.

[10] Hervik, S. 2010 Negatively curved left-invariant metrics on Lie groups. Preprint arXiv:1002.2106.

[11] Hewitt, C. G. and Wainwright, J. 1993 A dynamical systems approach to Bianchi cosmologies: orthogonal models of class B. Class. Quantum Grav. 10, 99-124.

[12] Horwood, J. T., Hancock, M. J., The, D. and Wainwright, J. 2003 Latetime asymptotic dynamics of Bianchi VIII cosmologies. Class. Quantum Grav. 20, 1757-1778.

[13] Lee, H. 2004 Asymptotic behaviour of the Einstein-Vlasov system with a positive cosmological constant. Math. Proc. Camb. Phil. Soc. 137, 495-509.

[14] Lin, X. F. and Wald, R. M. 1990 Proof of the closed universe recollapse conjecture. Phys. Rev. D41, 2444-2448.

[15] Patera, J., Sharp, R. T., Winternitz, P. and Zassenhaus, H. 1976 Invariants of real low dimension Lie algebras. J. Math. Phys. 17, 986-993. 
[16] Perko, L. 2001 Differential equations and dynamical systems. Springer, Berlin.

[17] Rendall, A. D. 1995 Global properties of locally spatially homogeneous cosmological models with matter. Math. Proc. Camb. Phil. Soc. 118, 145164 .

[18] Rendall, A. D. 2008 Partial differential equations in general relativity. Oxford University Press, Oxford.

[19] Ringström, H. 2001 The Bianchi IX attractor. Ann. H. Poincaré 2, 405-500.

[20] Ringström, H. 2001 The future asymptotics of Bianchi VIII vacuum solutions. Class. Quantum Grav. 18, 3791-3824.

[21] Ringström, H. 2004 On a wave map arising in general relativity. Commun. Pure Appl. Math. 57, 657-703.

[22] Varadarajan, V. S. 1984 Lie groups, Lie algebras and their representations.

[23] Wainwright, J. and Hsu, L. 1989 A dynamical systems approach to Bianchi cosmologies: orthogonal models of class A. Class. Quantum Grav. 6, 14091431.

[24] Wald, R. M. 1983 Asymptotic behaviour of homogeneous cosmological models in the presence of a positive cosmological constant. Phys. Rev. D28, $2118-2120$. 